\title{
Construction of fibronectin conditional gene knock-out mice and the effect of fibronectin gene knockout on hematopoietic, biochemical and immune parameters in mice
}

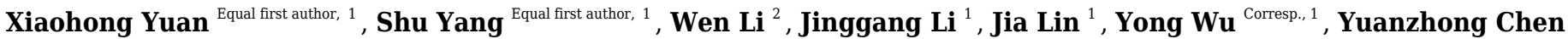 \\ Corresp. 1 \\ 1 Insitute of Hematology, Fujian Medical University Union Hospital, Fuzhou, Fujian, China \\ 2 Department of Pathology, Fujian Medical University Union Hospital, Fuzhou, Fujian, China \\ Corresponding Authors: Yong Wu, Yuanzhong Chen \\ Email address: Wuyong9195@126.com, chenyz@fjmu.edu.cn
}

Fibronectin (FN) is a multi-functional glycoprotein that primarily acts as a cell adhesion molecule and tethers cells to the extra cellular matrix. In order to clarify the effect of FN deficiency on hematopoiesis, biochemical and immune parameters in mice. We constructed a tamoxifen-induced conditional (cre-loxp system) fibronectin knock-out (FnKO) mouse model on a C57BL/6 background, and monitored their behavior, fertility, histological, hematopoietic, biochemical and immunological indices. We found that the Fn KO mice had reduced fertility, high platelet counts, smaller bone marrow megakaryocytes and looser attachment between the hepatocyte and vascular endothelial junctions compared to the wild type (WT) mice. In contrast, the behavior, hematological counts, serum biochemical indices and vital organ histology were similar in both Fn KO and WT mice. This model will greatly help in elucidating the role of FN in immune-related diseases in future. 
fibronectin gene knockout on hematopoietic, biochemical and immune parameters in mice

3 Xiaohong Yuan ${ }^{1 \#}$, Shu Yang ${ }^{1 \#}$, Wen $\mathrm{Li}^{2}$, Jinggang $\mathrm{Li}^{1}$, Jia Lin ${ }^{1}$, Yong Wu ${ }^{1 *}$, Yuanzhong Chen ${ }^{1 *}$

$4 \quad{ }^{1}$ Fujian Insitute of Hematology, Fujian Provincial Key Laboratory on Hematology, Fujian

5 Medical University Union Hospital, Fuzhou, Fujian, China.

$6 \quad{ }^{2}$ Department of Pathology, Fujian Medical University Union Hospital, Fuzhou, Fujian, China.

7 *Corresponding author: Yuanzhong Chen, Insitute of Hematology, Chairman of the Fujian

8 Medical University Union Hospital, Xinquan Road 29, Gulou District, Fuzhou City, Fujian

9 Province, China. Email: chenyz@,fjmu.edu.cn

$10 *$ Co- corresponding author: Yong Wu, Insitute of Hematology, Professor of Internal Medicine in

11 Fujian Medical University Union Hospital, Xinquan Road 29, Gulou District, Fuzhou City,

12 Fujian Province, China. Email: Wuyong9195@126.com

\section{Abstract:}

14 Fibronectin $(\mathrm{FN})$ is a multi-functional glycoprotein that primarily acts as a cell adhesion 15 molecule and tethers cells to the extra cellular matrix. In order to clarify the effect of FN 16 deficiency on hematopoiesis, biochemical and immune parameters in mice. We constructed a 17 tamoxifen-induced conditional (cre-loxp system) fibronectin knock-out (FnKO) mouse model on 18 a C57BL/6 background, and monitored their behavior, fertility, histological, hematopoietic, 19 biochemical and immunological indices. We found that the Fn KO mice had reduced fertility, 20 high platelet counts, smaller bone marrow megakaryocytes and looser attachment between the 21 hepatocyte and vascular endothelial junctions compared to the wild type (WT) mice. In contrast, 
22 the behavior, hematological counts, serum biochemical indices and vital organ histology were

23 similar in both Fn KO and WT mice. This model will greatly help in elucidating the role of FN in

24 immune-related diseases in future.

25 Keywords: Fibronectin gene knockout, hematopoietic function, immune parameters, fertility

\section{Introduction}

Fibronectin (FN) is a cell adhesion glycoprotein that was first discovered in plasma by Morrison (Morrison et al. 1948), and it was characterized by Mosesson in 1970 in plasma(Mosesson \& Umfleet 1970). It was called CIG (cold-insoluble globulin) before being named as Fibronectin. Subsequent studies have found that FN is widespread in the intercellular medium and even on the surface of cancer cells(Hynes 1985). FNs are generated by alternative splicing from a single gene, and it exists in two different forms, one form is cellular FN (cFN), which contains, depending on the tissue, variable proportions of the alternatively spliced exons coding for the extra domains A and B (EDA, EDB). The other form is plasma FN (pFN), which lacks EDA and EDB. pFN is synthesized by hepatocytes and released into the circulation where it remains soluble(White et al. 2008). FN has multiple functional domains that can bind to integrin, collagen, fibrin and heparin, and participate in cell adhesion, migration, proliferation and differentiation(Hynes 2012; Mao \& Schwarzbauer 2005; Pankov 2002). More and more studies have demonstrated that FN plays an important role in assessement the severity of severe infection and sepsis(Lemańska-Perek \& Adamik 2019; Ruiz Martin et al. 2004).

At present, FN knockout animal models are urgently needed to study the role of FN in infection and sepsis. In order to study the physiological function of $\mathrm{FN}$, scientists began to try to construct 
43 Fn gene knockout mice in the 1990s. At first, the researchers found that Fn gene knockout at

44 embryonic level was fatal because the development of cardiovascular system in mesoderm was

45 blocked(George et al. 1993). In 2001, Fässler et al. established a conditional Fn gene knockout

46 (KO) mouse model based on the cyclo-recombinase (cre-loxp) system(Sakai et al. 2001), which

47 was induced by the intraperitoneal injection of polyI-polyC. However, the latter can promote interferon (IFN) production in body and thus potentially affect the immune system, making this model unsuitable for studying the hematopoietic and immune-related functions of FN. The

Fässler group also used albumin-cre mice in later work, they analyzed the role of FN in many different pathophysiological states such as atherosclerosis and skeletal muscle regeneration and many others(Konstandin et al. 2013; Rohwedder et al. 2012). However, no reports are available so far on the effects of Fn gene knockout on the vital organs, hematopoiesis, biochemical indices and immune status of mice.

To clarify the effects of FN on the above, we constructed a tamoxifen-induced conditional Fn

KO model against the C57BL/6 background using the cre-loxp system, where in the first exon of the Fn gene was modified by loxp. We achieved a knockout efficiency was $95 \%$, as determined by the decrease in the levels of circulating plasma FN after tamoxifen induction. In addition, the Fn KO mice also showed significantly lower in situ expression of FN in the vital organs compared to the wild type (WT) mice. Although the hematopoietic, biochemical and histological indices of the Fn KO and WT mice were largely similar, the former differed in some aspects, our findings can help elucidate the functions of FN. 


\section{Materials \& Methods}

\section{Loxp modification of mouse Fn 1 gene}

Target gene name (MGI number): Fn1 (95566), Fn transcript for the knockout protocol (Ensembl number): Fn1-001(ENSMUST00000055226), Flox-targeted Fn exons: exon 1. Using the principle of homologous recombination, the Fn1 gene was subjected to flox modification by means of embryonic stem (ES) cell targeting. The brief process is as follows: Bacterial artificial chromosome (BAC) clones containing Fn gene are purchased from Sanger Institute (UK). The ES cell targeting vector was constructed by the method of ET-clone. The vector comprises a $4 \mathrm{~kb}$ 5' homology arm, a $0.5 \mathrm{~kb}$ flox region, a PGK-Neo-polyA, a 4 kb 3' homology arm, and a MC1TK-polyA negative selection marker. After the vector is linearized, it is electrotransfected into C57*129 cells. After screening with G418 and Ganc drugs, hundreds of resistant clones were obtained, and positive clones with correct homologous recombination were identified by long fragment PCR. The positive ES cell clone was amplified and injected into the blastocyst of C57BL/6J mice to obtain a chimeric mouse. High proportion of chimeric mice were mated with Flp mice to obtain positive F1 mice (This part of the experiment was completed with the assistance of Southern Model Biotechnology). The Fn vector plasmid construction model is shown in Figure 1.

\section{Animals}

Specific pathogen free (SPF) male and female C57BL/6J mice (8 to 12 weeks old, weighing 20 22g) were purchased from Shanghai SLAC LABORATORY ANIMAL COMPANY. The Fn loxp $+/+($ Fn Flox $)$ mice were generated in our lab with technical support from the Shanghai 
84 Biomodel Organism Science \& Technology Development. UBC-cre/ERT2 mice were purchased

85

86

87

from Shanghai Biomodel Organism Science \& Technology Development. The mice were housed

five to six per cage under specific pathogen-free conditions, and the animal housing included a controlled light and dark cycle (12h: $12 \mathrm{~h}$ ), ad libitum food and ultra filtered water, $50-55 \%$ humidity, ventilated caging systems and standardized environmental enrichment. Except that the mice used to observe the lifespan were waiting for natural death, all the other surviving mice were euthanized by inhalation of an overdose of sevoflurane after the end of the experiment. All animals were handled in strict accordance with good animal practice as defined by the National Regulations for the Administration of Experimental Animals of China and the National Guidelines for Experimental Animal Welfare of China. Animal protocols and experimental procedures were also approved by the Institutional Animal Care and Use Committee of the University of Fujian Medical University (Ethical Approval Number: 2017-0135).

\section{The propagation of mice}

Fn Loxp+ F1 mice were backcrossed with wild C57BL/6J mice, and the offspring were either self-mating or mating with UBC-cre/ERT2 mice to obtain progeny Fn Loxp+/+ and Fn Loxp + Cre + mice. Fn Loxp $+/+$ mice were mated with Fn Loxp + Cre + mice to obtain progeny Fn Loxp $+/+$ Cre + mice finally. WT, Fn Loxp + and Fn Loxp $+/+$ Cre + (Fn KO) mice of the same age and born in the same litter were selected for the study. The mice began intraperitoneal injection of tamoxifen $0.2 \mathrm{mg} / \mathrm{g}$ body weight (corn oil with tamoxifen $10 \mathrm{mg} / \mathrm{ml}$ ) at 4 weeks age for 2 times, interval 2 days. The dietary and defecation habits, changes in body weight and fur, and the fertility status of the three groups mice were observed for 12 months. 
105

106

107

108

109

110

111

112

113

114

115

116

117

118

119

120

121

122

123

124

125

\section{Routine blood, biochemical and histological examination}

WT, Fn Loxp and Fn KO mice (4 male and 4 females per group) weighing 20-22 g were randomly selected 4 weeks after the tamoxifen injection, and anaesthetized by the intraperitoneal injection of $100-120 \mu 10.1 \%$ pentobarbital. Blood was collected from the canthal vein, and subjected to routine cytological and biochemical tests. The chest and abdominal wall were dissected along the midline, and the liver, spleen, kidney, heart, lungs, femur and brain were extracted. All tissues were fixed overnight in 10\% formalin, embedded in paraffin, sectioned and stained with hematoxylin and eosin as per standard protocols. In addition, the liver and peritoneal blood vessels were also processed for transmission electron microscopy (TEM).

\section{Flow cytometry}

The bone marrows were flushed out, and the spleen was homogenized in PBS. The different homogenates were filtered through a $75 \mu \mathrm{m}$ mesh after erythrocyte lysis. The Fc receptors on the mononuclear cells were blocked with anti-mouse CD16/32 (BD Pharmingen) antibodies for 10 minutes, and the cells were then stained with the fluorochrome-conjugated antibodies for 30 minutes at $4^{\circ} \mathrm{C}$ in the dark. After washing twice with PBS, the cells were acquired on a BD FACS Verse flow cytometer (BD Bioscience) and the data was analyzed with FlowJo 10 software.

\section{Reagents}

Tamoxifen (T5648) was purchased from Sigma-Aldrich. The antibodies (Abs) against mouse CD11b-PE, CD4-PE, CD3-APC-CyTM7, CD8a-FITC, CD220-FITC and CD25-FITC, and the isotype control were from $\mathrm{BD}$ Pharmingen. The cell staining buffer was purchased from 
126

127 and the antibody (ab23750) against FN were from Abcam.

128 Statistical Analysis

129 All data were analyzed using GraphPad Prism6 software. Two groups were compared by Student 130

eBiolegend, and PBS and erythrocyte lysis buffer were from Hyclone. The ELISA kit (ab210967)

\begin{abstract}
$\mathrm{t}$ test, and $\mathrm{P}<0.05$ was considered statistically significant. Proportions for categorical variables
\end{abstract} were compared using the $\chi 2$ test. Values are presented as the mean or mean $\pm \mathrm{SD}$.

\section{Results}

\section{Successful generation of Fn gene conditional knockout mice}

Identification and linearization of the Fn1 gene targeting vector, and the results of Hind III digestion, the theoretical band size is $9.8 \mathrm{~kb}, 5.8 \mathrm{~kb}$ (810 bp band is too small to shown) (Figure

2A). A total of $144 \mathrm{ES}$ cells resistant clones were obtained, after sequencing the above cloning

PCR products, a total of 10 correct homologous recombination positive ES cell clones were obtained. And finally, we got three mice with homologous recombination-positive F1 mice, which were number 7,14 , and 16 respectively, which were confirmed to be Loxp-positive by sequencing. The long-segment PCR identification electrophoresis 5' homology arm (5'arm) and 3' homology arm (3'arm) are shown in Figure 2B. The 5 'arm homologous recombination positive clones amplified $4.2 \mathrm{~kb}$ and $5.6 \mathrm{~kb}$ fragments, while the negative clones amplified only $5.6 \mathrm{~kb}$ fragments. 3 'arm homologous recombination positive cloning should amplify the fragment of $4.6 \mathrm{~kb}$ and $5.9 \mathrm{~kb}$, while the negative cloning could only amplify the fragment of 5.9kb. Fn loxP $+/+\mathrm{Cre}+$ mice were induced by tamoxifen peritoneal injection for 1 week. The 
146 PCR analysis suggested that the knockout efficiency was up to $99 \%$ in gene level from liver

147 (Figure 2C). The efficiency of FN knockout was determined by analyzing the levels of the

148 protein in plasma and liver by ELISA and Western blotting, and a 95\% reduction was observed

149 (Figure 2D). In addition, the in situ levels of FN in the vital organs of the Fn KO mice were 150 significantly reduced compared to that in the WT mice, FN immunohistochemical and immunofluorescence staining of liver and kidney indicated that FN expression was decreased significantly in Fn KO mice (Figure 2E).

\section{Reproductive ability of Fn KO mice was significantly reduced}

No significant changes were seen in the body weight, fur and other physiological indices of three groups of mice even after 12 months of tamoxifen induction. However, the fertility of female Fn

KO mice was significantly reduced, the average number of pregnancies per year was significantly reduced, with a litter size of only 1-2, and the neonates showed high rates of

malformation and mortality in Fn KO mice, $p<0.0001$ (Table 1). The malformed mouse offspring we observed were mainly spinal dysplasia and slender limbs.

\section{Changes of Hematopoietic, Biochemical and Immune Parameters in Fn KO Mice}

We found except that platelet counts increased in Fn KO mice $(\mathrm{P}<0.05)$, the whole blood and serum analysis did not reveal any significant differences between the Fn $\mathrm{KO}$ and other two groups of mice in terms of cytological and biochemical indices (Tables 2 and 3).

Flow cytometry analysis of peripheral blood, bone marrow and spleen cells also did not show any aberration in the proportions of the lymphocyte, NK cell and monocyte subsets in the Fn KO mice, which were overall similar to that of the other two groups of mice (Figure 3A). 
167 Histological and electron microscopic observation of important organs in Fn KO mice

168 No major histological changes were observed in the heart, liver, spleen, lung, kidney and brain of 169 the Fn KO mice relative to the WT mice, and the lymph node distribution was also normal. 170 However, compared to the WT mice, the megakaryocytes in the bone marrow of Fn KO mice 171 were smaller with decreased nuclear lobulation, but their proportion was higher (Figure 3B).

172 TEM of the liver sections of WT mice showed tight and clear-edged intercellular links between 173 the liver parenchyma and peritoneal vascular endothelial cells (VECs), while the liver parenchyma in the Fn KO mice was loose with blurred cell edges and increased space between the VECs (Figure 3C).

Discussion

177 Gene knockout animal models are a powerful tool to study the physiological functions of the respective genes. The Fn knockout model was first devised in the 1990s, but was embryonically lethal due to abnormal development of cardiovascular system in the mesoderm(George et al. 1993). In 2001, Fässler et al. established a conditional Fn knockout using the Cre-Loxp system, which reduced the plasma FN levels to less than 5\% of the physiological value. This model helped elucidate the role of $\mathrm{FN}$ in coagulation, tissue repair, atherosclerosis and stroke( $\mathrm{Ni}$ et al. 2003; Rohwedder et al. 2012; Sakai et al. 2001). However, the Cre-induced knockout was dependent on interferon, which was stimulated in vivo by intraperitoneal injection of polyI-polyC.

High levels of interferon can potentially affect the immune function, making this model unsuitable for studying the role of FN in hematopoietic and immune-related functions. Although 
188 189 190

different pathophysiological states such as atherosclerosis and skeletal muscle regeneration. No reports are available so far on the effects of Fn gene knockout on the vital organs, hematopoiesis, biochemical indices and immune status of mice.

We developed a conditional knockout model based on tamoxifen induction, and validated absence of FN in the plasma as well as the solid tissues after 4 weeks of induction. Fn knockout had no significant effect on the food intake, defecation frequency, fur color and body weight, although the fecundity of the female Fn KO mice and the viability of the neonates were significantly reduced compared to the WT mice. Previous studies have documented that FN is crucial in the development of blood vessels during embryogenesis(George et al. 1993; Kumra et al. 2018). It has also been reported that Fank1 (Fibronectin Type3 domain)-knockout did not cause changes in sperm quality or quantity in male mice(Zhang et al. 2019), however, the effect of FN deficiency on the fertility of male mice still need to be further studied.

We found there were no substantial differences between the Fn KO and WT mice in terms of peripheral blood counts, serum biochemical indices, tissue architecture, and the hematological cell subtypes in bone marrow, spleen and peripheral blood. However, we found the platelet counts increased in Fn KO mice, but the megakaryocytes in the bone marrow of Fn KO mice were noticeably smaller and showed decreased nuclear lobulation. It has been reported that FN can regulate and impact megakaryocyte behavior differently during their differentiation(Malara et al. 2014; Malara et al. 2011). It was recently reported that fibronectin EDA isoform can sustain megakaryocyte expansion and participate in the inflammatory process of myelofibrosis(Malara et al. 2019; Matsuura et al. 2020). However, the mechanism by which FN 
209

210

211

212

213 loss of cellular type FN, which should be verified in future studies.

\section{Conclusion}

215 To summarize, we established a tamoxifen-induced conditional Fn gene knockout mouse model, 216

220

221

This work was supported by the Special Financial Found of Fujian Provincial Department of 
230 Science and Technology (Min 2015-1297, Major Innovation Projects in Fujian Province),

231 Construction Project of Fujian Medical Center of Hematology (Min 2017-04) and National and

232 Fujian Provincial Key Clinical Specialty Discipline Construction Program of China.

\section{Author Contributions}

Drs. Yuan and Yang conceived and performed animal experiments, analysed and interpreted data,

and drafted the paper. Wen $\mathrm{Li}$ and Jinggang $\mathrm{Li}$ participated in performing morphological

examination of mouse tissue sections. Lin assisted in the reproduction and identification of the

mice. Dr. Chen and $\mathrm{Wu}$ are the project leaders, designed experiments, participating in drafting

the article, and approved the final version of the article. "Drs. Yuan and Yang contributed equally

to this work.

\section{References}

George EL, Georges-Labouesse EN, Patel-King RS, Rayburn H, and Hynes RO. 1993. Defects in mesoderm, neural tube and vascular development in mouse embryos lacking fibronectin. Development 119:1079-1091.

Hynes R. 1985. Molecular biology of fibronectin. Annu Rev Cell Biol 1:67-90. 10.1146/annurev.cb.01.110185.000435

Hynes RO. 2012. The evolution of metazoan extracellular matrix. J Cell Biol 196:671-679. 10.1083/jcb.201109041

Konstandin MH, Toko H, Gastelum GM, Quijada P, De La Torre A, Quintana M, Collins B, Din S, Avitabile D, Volkers M, Gude N, Fassler R, and Sussman MA. 2013. Fibronectin is essential for reparative cardiac progenitor cell response after myocardial infarction. Circ Res 113:115-125. 10.1161/CIRCRESAHA.113.301152

Kumra H, Sabatier L, Hassan A, Sakai T, Mosher DF, Brinckmann J, and Reinhardt DP. 2018. Roles of fibronectin isoforms in neonatal vascular development and matrix integrity. PLoS Biol 16:e2004812. 10.1371/journal.pbio.2004812

Lemańska-Perek A, and Adamik B. 2019. Fibronectin and its soluble EDA-FN isoform as biomarkers for inflammation and sepsis. Adv Clin Exp Med 28:1561-1567. 10.17219/acem/104531

Malara A, Currao M, Gruppi C, Celesti G, Viarengo G, Buracchi C, Laghi L, Kaplan DL, and Balduini A. 2014. Megakaryocytes contribute to the bone marrow-matrix environment by expressing fibronectin, type IV collagen, and laminin. Stem Cells 32:926-937. 10.1002/stem.1626

Malara A, Gruppi C, Abbonante V, Cattaneo D, De Marco L, Massa M, lurlo A, Gianelli U, Balduini CL, Tira ME, Muro 
AF, Chauhan AK, Rosti V, Barosi G, and Balduini A. 2019. EDA fibronectin-TLR4 axis sustains megakaryocyte expansion and inflammation in bone marrow fibrosis. J Exp Med 216:587-604. 10.1084/jem.20181074

Malara A, Gruppi C, Rebuzzini P, Visai L, Perotti C, Moratti R, Balduini C, Tira ME, and Balduini A. 2011. Megakaryocyte-matrix interaction within bone marrow: new roles for fibronectin and factor XIII-A. Blood 117:2476-2483. 10.1182/blood-2010-06-288795

Mao Y, and Schwarzbauer JE. 2005. Fibronectin fibrillogenesis, a cell-mediated matrix assembly process. Matrix Biology 24:389-399. 10.1016/j.matbio.2005.06.008

Matsuura S, Thompson CR, Ng SK, Ward CM, Karagianni A, Mazzeo C, Malara A, Balduini A, and Ravid K. 2020. Adhesion to Fibronectin via $\alpha 5 \beta 1$ Integrin Supports Expansion of Megakaryocyte Lineage in Primary Myelofibrosis. Blood. 10.1182/blood.2019004230

Morrison PR, Edsall JT, and Miller SG. 1948. Preparation and properties of serum and plasma proteins; the separation of purified fibrinogen from fraction I of human plasma. J Am Chem Soc 70:3103-3108. 10.1021/ja01189a080

Mosesson MW, and Umfleet RA. 1970. The Cold-insoluble Globulin of Human Plasma. J Biol Chem 245:5736-5738.

Ni H, Yuen PS, Papalia JM, Trevithick JE, Sakai T, Fassler R, Hynes RO, and Wagner DD. 2003. Plasma fibronectin promotes thrombus growth and stability in injured arterioles. Proc Natl Acad Sci U S A 100:2415-2419. 10.1073/pnas.2628067100

Pankov R. 2002. Fibronectin at a glance. Journal of Cell Science 115:3861-3863. 10.1242/jcs.00059

Rohwedder I, Montanez E, Beckmann K, Bengtsson E, Duner P, Nilsson J, Soehnlein O, and Fassler R. 2012. Plasma fibronectin deficiency impedes atherosclerosis progression and fibrous cap formation. EMBO Mol Med 4:564-576. 10.1002/emmm.201200237

Ruiz Martin G, Prieto Prieto J, Veiga de Cabo J, Gomez Lus L, Barberan J, Gonzalez Landa JM, and Fernandez C. 2004. Plasma fibronectin as a marker of sepsis. Int J Infect Dis 8:236-243. 10.1016/j.ijid.2003.10.005

Sakai T, Johnson KJ, Murozono M, Sakai K, Magnuson MA, Wieloch T, Cronberg T, Isshiki A, Erickson HP, and Fassler R. 2001. Plasma fibronectin supports neuronal survival and reduces brain injury following transient focal cerebral ischemia but is not essential for skin-wound healing and hemostasis. Nat Med 7:324-330. $10.1038 / 85471$

White ES, Baralle FE, and Muro AF. 2008. New insights into form and function of fibronectin splice variants. $J$ Pathol 216:1-14. 10.1002/path.2388

Zhang J, Zhang X, Zhang Y, Zeng W, Zhao S, and Liu M. 2019. Normal spermatogenesis in Fank1 (fibronectin type 3 and ankyrin repeat domains 1) mutant mice. PeerJ 7:e6827. 10.7717/peerj.6827

Peer] reviewing PDF | (2020:06:49574:1:0:NEW 16 Sep 2020) 


\section{Table $\mathbf{1}$ (on next page)}

The fertility of three groups of female mice (Mean \pm SD) 
1 Table 1: The fertility of three groups of female mice (Mean \pm SD)

\begin{tabular}{llll}
\hline Parameter & WT $(\mathrm{n}=6)$ & Fn Loxp $(\mathrm{n}=6)$ & Fn KO $(\mathrm{n}=6)$ \\
\hline Average number of pregnancies per year & $8.3 \pm 1.1$ & $8.0 \pm 1.0$ & $6.4 \pm 1.2^{*}$ \\
The average number of births per litter & $7.8 \pm 0.54$ & $7.4 \pm 0.58$ & $1.7 \pm 0.43^{* * * *}$ \\
Total breeding population per year & 388 & 366 & 63 \\
Survival rate of mice \% $(\mathrm{n})$ & $96.9(376)$ & $95.4(349)$ & $34.9(22)^{* * * *}$ \\
\hline
\end{tabular}

2

$* \mathrm{P}<0.05, * * * * \mathrm{P}<0.0001$

3 
Table 2 (on next page)

Hematological counts of three groups of mice (Mean \pm SD) 
1 Table 2: Hematological counts of three groups of mice (Mean \pm SD)

\begin{tabular}{llll}
\hline Parameter & WT $(\mathrm{n}=8)$ & Fn Loxp $(\mathrm{n}=8)$ & Fn KO $(\mathrm{n}=8)$ \\
\hline Leukocytes $\left(10^{9} / \mathrm{L}\right)$ & $8.64 \pm 1.32$ & $8.94 \pm 1.43$ & $9.96 \pm 1.62$ \\
Neutrophils $\left(10^{9} / \mathrm{L}\right)$ & $2.37 \pm 1.64$ & $2.42 \pm 1.58$ & $3.21 \pm 1.52$ \\
Lymphocytes $\left(10^{9} / \mathrm{L}\right)$ & $6.02 \pm 1.86$ & $6.21 \pm 1.80$ & $7.16 \pm 1.64$ \\
Monocytes $\left(10^{9} / \mathrm{L}\right)$ & $0.66 \pm 0.35$ & $0.65 \pm 0.30$ & $0.52 \pm 0.32$ \\
RBC $\left(10^{12} / \mathrm{L}\right)$ & $9.16 \pm 0.62$ & $9.09 \pm 0.69$ & $8.90 \pm 0.86$ \\
Hgb $(\mathrm{g} / \mathrm{L})$ & $146.2 \pm 6.04$ & $143.9 \pm 6.98$ & $138.6 \pm 10.6$ \\
PCV $(\%)$ & $45.6 \pm 3.55$ & $45.26 \pm 3.81$ & $42.2 \pm 3.20$ \\
Platelet $\left(10^{9} / \mathrm{L}\right)$ & $726 \pm 168.4$ & $738 \pm 173.6$ & $923 \pm 204^{*}$ \\
\hline
\end{tabular}

$2 * \mathrm{P}<0.05$

3 


\section{Table 3(on next page)}

Serum biochemical indices of three groups of mice (Mean \pm SD) 
1 Table 3: Serum biochemical indices of three groups of mice (Mean \pm SD)

\begin{tabular}{llll}
\hline Parameter & WT $(\mathrm{n}=8)$ & Fn Loxp $(\mathrm{n}=8)$ & Fn KO $(\mathrm{n}=8)$ \\
\hline $\mathrm{TP}(\mathrm{g} / \mathrm{L})$ & $58.6 \pm 2.46$ & $58.9 \pm 2.08$ & $57.4 \pm 1.82$ \\
$\mathrm{ALB} \quad(\mathrm{g} / \mathrm{L})$ & $22.6 \pm 5.64$ & $21.7 \pm 5.19$ & $18.62 \pm 4.50$ \\
$\mathrm{AST} \quad(\mathrm{IU} / \mathrm{L})$ & $58.2 \pm 6.20$ & $62.6 \pm 7.36$ & $86.4 \pm 10.6$ \\
$\mathrm{ALT}(\mathrm{IU} / \mathrm{L})$ & $189.4 \pm 11.3$ & $188.2 \pm 13.4$ & $196.6 \pm 12.6$ \\
$\mathrm{TG}(\mathrm{mmol} / \mathrm{L})$ & $0.88 \pm 0.46$ & $0.95 \pm 0.57$ & $1.04 \pm 0.64$ \\
$\mathrm{CHOL}(\mathrm{mmol} / \mathrm{L})$ & $2.58 \pm 0.22$ & $2.62 \pm 0.19$ & $2.50 \pm 0.15$ \\
$\mathrm{UREA} \quad(\mathrm{mmol} / \mathrm{L})$ & $9.14 \pm 0.72$ & $9.21 \pm 0.78$ & $8.35 \pm 0.86$ \\
$\mathrm{CREA} \quad(\mu \mathrm{mol} / \mathrm{L})$ & $29.8 \pm 4.60$ & $29.4 \pm 5.68$ & $31.0 \pm 7.13$ \\
\hline
\end{tabular}

2 
Figure 1

Target vector plasmid map

Fn1 target vector plasmid map

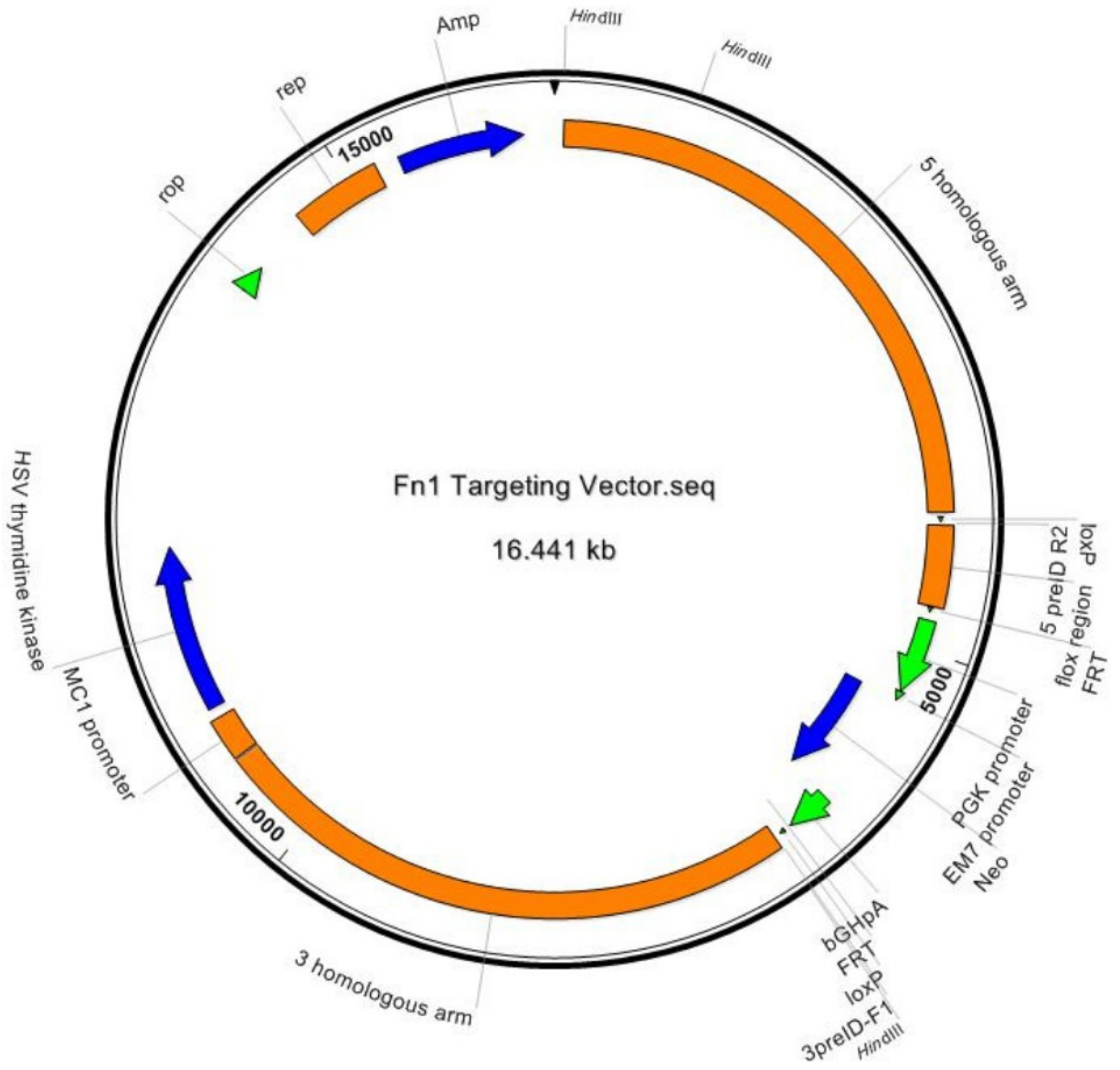




\section{Figure 2}

Successful generation of Fn gene conditional knockout mice

(A) Identification and linearization of the Fn1 gene targeting vector, and the results of Hind III digestion, the theoretical band size is $9.8 \mathrm{~kb}, 5.8 \mathrm{~kb}$ (810 bp band is too small to hown). (B) Electrophoresis map of $5^{\prime}$ homology arm (5'arm) and 3' homology arm (3'arm) PCR identification of F1 mice (recombinant negative can only clone a single fragment). (C) The PCR product of the genome inserted into LoxP was $1.4 \mathrm{~kb}$, the PCR product of KO was $0.7 \mathrm{~kb}$, and the PCR product of WT mouse was $1.2 \mathrm{~kb}$, electrophoresis grayscale graph showed that knockout efficiency of DNA was $99 \%$ in liver. (D) ELISA and Western blotting results showing $\mathrm{FN}$ levels in the plasma and liver of Fn KO mice at different durations post tamoxifen induction, the knockout efficiency was 95\%. (E) FN immunohistochemical and immunofluorescence staining of liver and kidney indicated that FN expression was decreased significantly in Fn KO mice. 
A

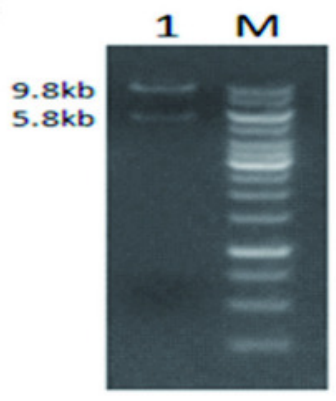

GeneRuler$1 \mathrm{~kb}$ DNA Ladder

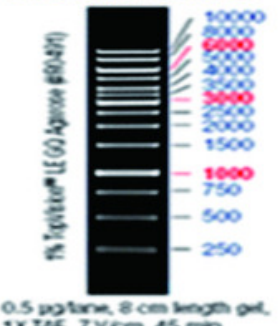

0.5 potire 8 cen mon on.
B
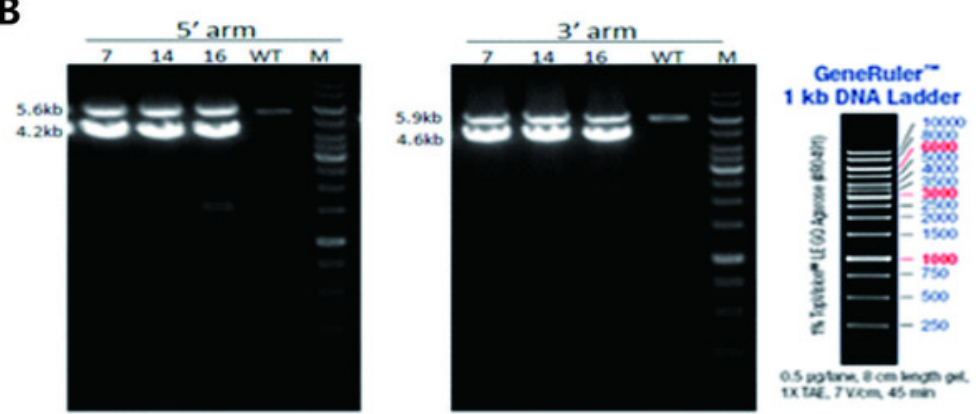

C

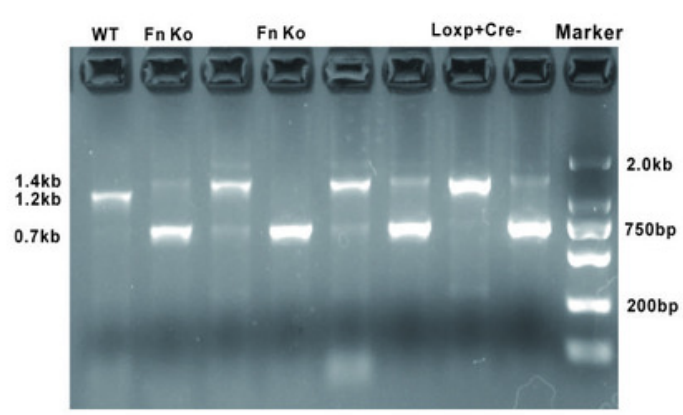

$\mathbf{E}$

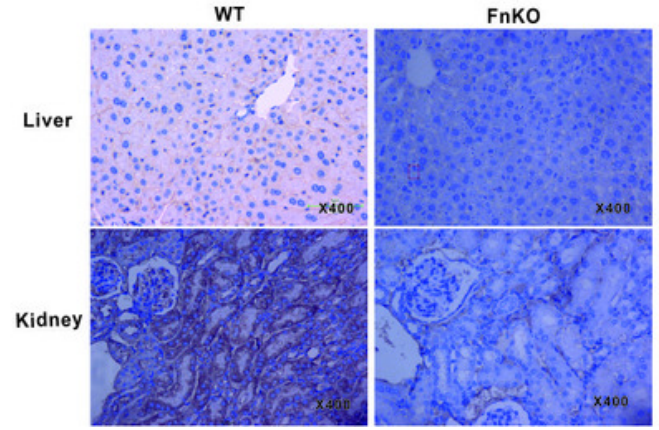

D
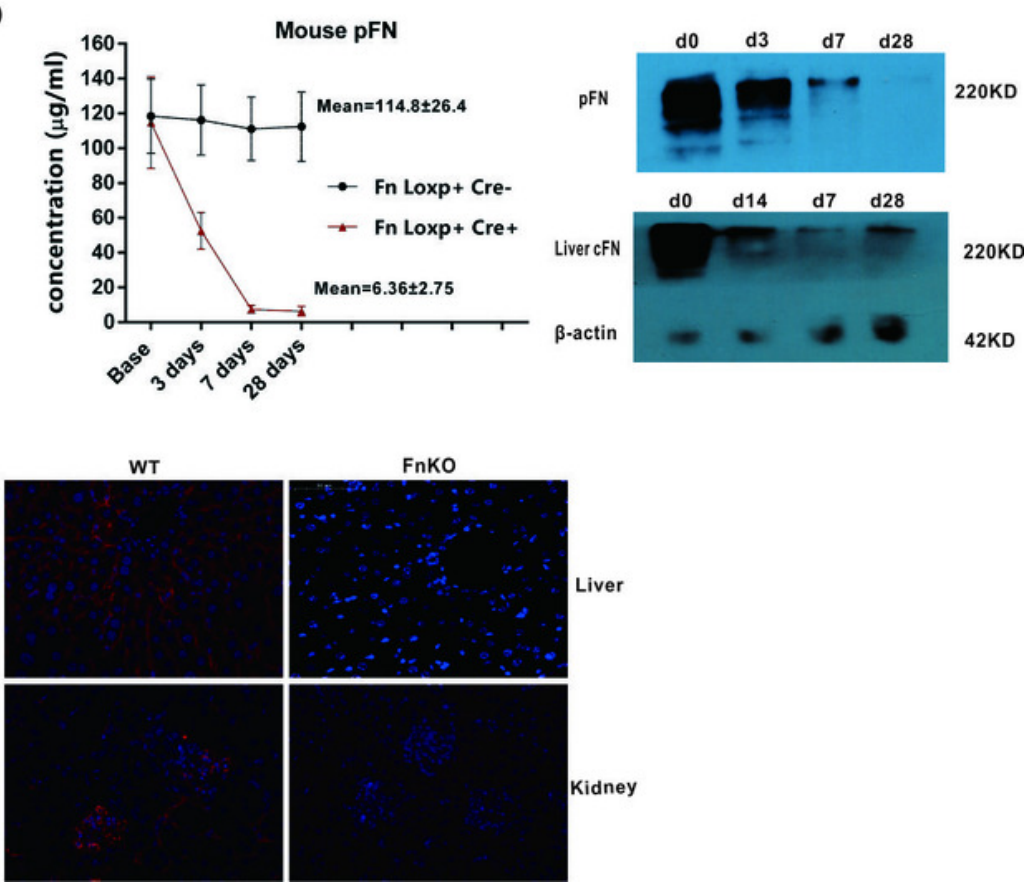

FnKo

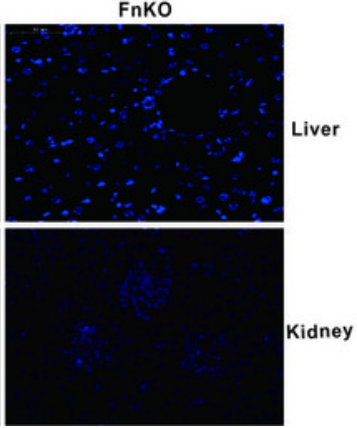




\section{Figure 3}

Observation of immune cell subsets, bone marrow morphology and vascular endothelial tissue in FnKO mice

(A) The proportion of different cell types in the peripheral blood, bone marrow and spleen were analyzed by Flow cytometry in Fn KO mice, compared with the normal reference value, no significant differences were found. (B) Representative H\&E stained images of the bone marrow megakaryocytes of Fn KO and WT mice, the megakaryocytes in the bone marrow of Fn KO mice were smaller with decreased nuclear lobulation. (C) Representative TEM images of the liver parenchyma and peritoneal vascular endothelial cells (VECs) of Fn KO and WT mice, the liver parenchyma in the Fn KO mice was loose with blurred cell edges and increased space between the VECs. 
A
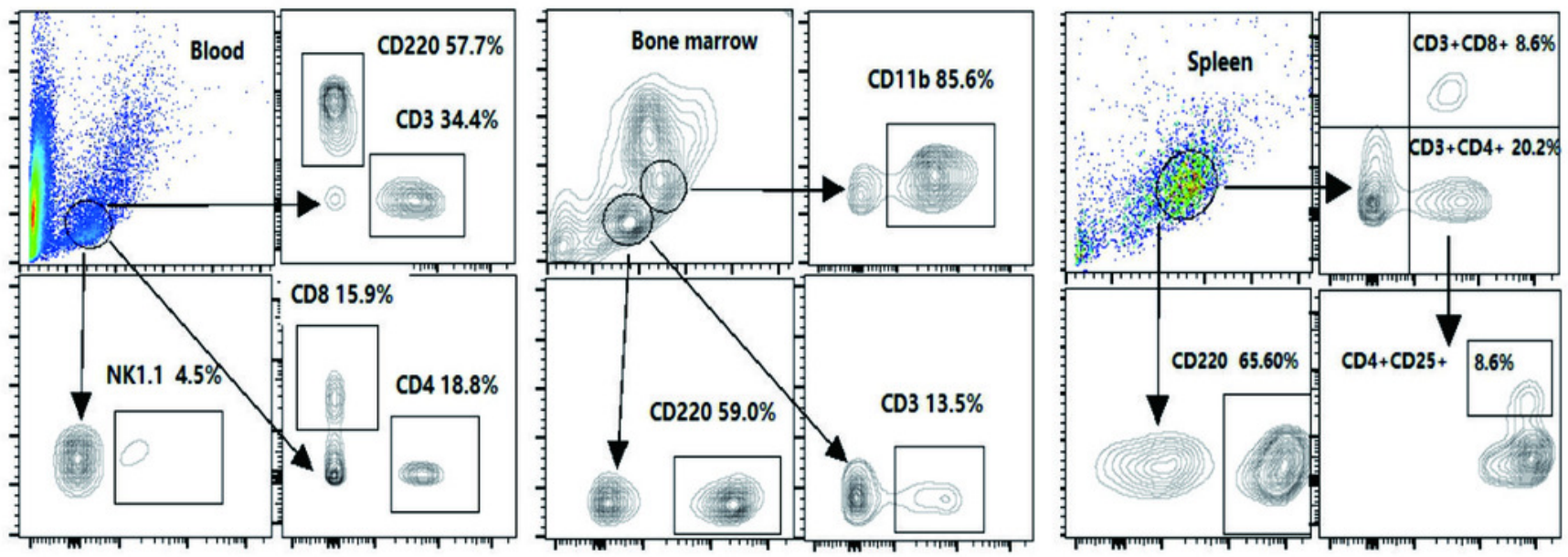

B

WT
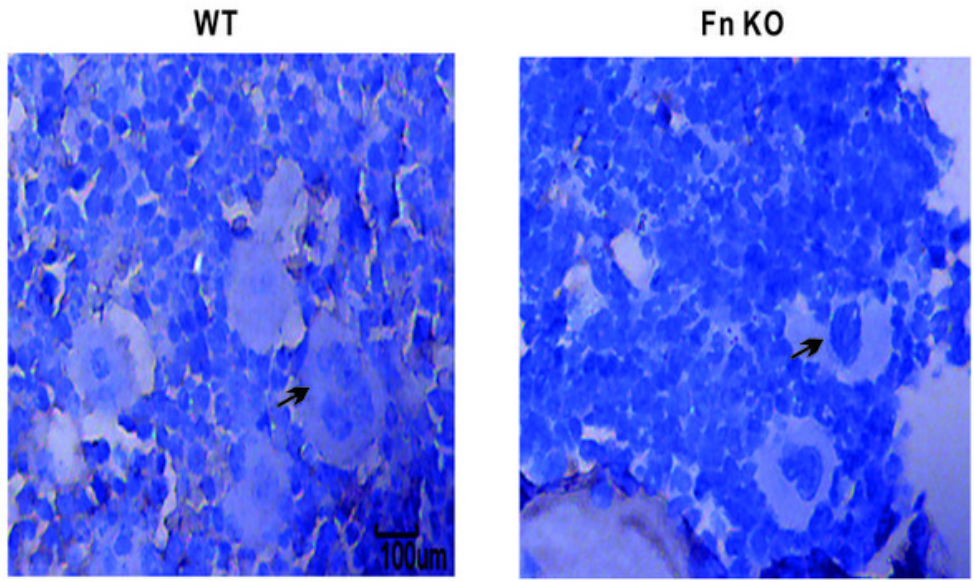

C

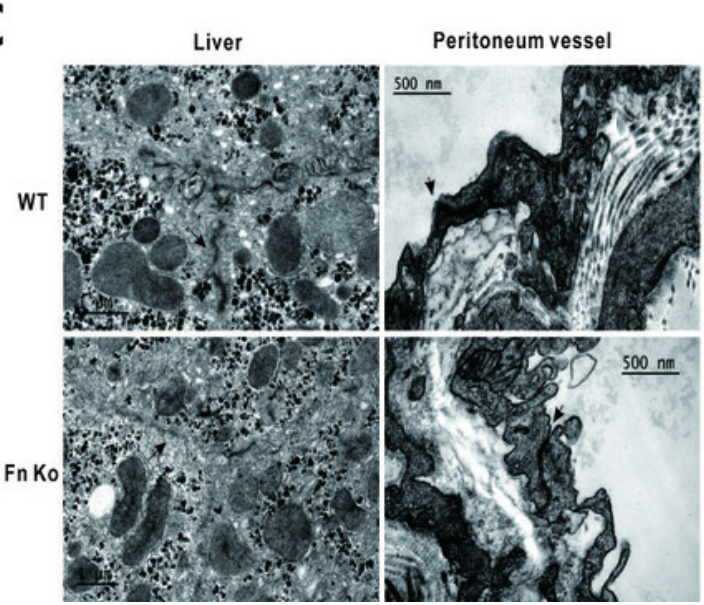

